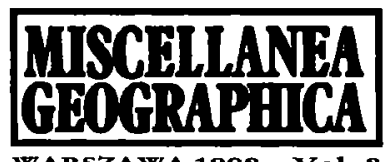

Katarzyna Wesołowska

\title{
VARIATIONS ANNUELLES DU NOMBRE DE JOURS D'ORAGE À VARSOVIE
}

Nous aimerions présenter ici le caractère cyclique et les tendances de variations du nombre de jours d'orage en même temps que déterminer les rapports entre ledit phénomène et l'activité du Soleil, les types de la circulation atmosphérique et la température de l'air, sur l'exemple de Varsovie.*

Les données de source ont été recueillies dans la station météorologique située à Varsovie dans l'aéroport d'Okęcie et se rapportent aux années 1951-1980. Pour la période de quatre mois (mai - août), on a désigné les cycles réels de l'activité solaire, ceux de la circulation atmosphérique selon la classification de Wangenheim-Girs (Girs, 1974; Boryczka et. al., 1997), de la température de l'air et du nombre de jours d'orage.

À Varsovie, tout comme dans tout le territoire de la Pologne, les orages se manifestent le plus souvent vers la fin du printemps, en été et au début de l'automne. Ainsi, dans la période qui nous intéresse il y avait 131 orages en mai, 178 - en juin, 190 - en juillet 153 - en août et 57 - en septembre.

Au cours des trois décennies en question (1951-1980), on a observé 800 jours d'orage ce qui fait env. 27 jours d'orage en moyenne par an. Parmi les années les plus orageuses, citons 1955 (38 orages), 1961 (35), 1963 (35), 1968 (33) et 1972 (33) tandis que les années 1953, 1973 et 1976 n'abondaient plutôt pas en ces phénomènes atmosphériques (respectivement 18,19 , et 15 orages).

La radiation directe du Soleil est la source principale de l'énergie thermique, indispensable aux processus météorologiques s'opérant sur la Terre. Ainsi, les spectres de l'oscillation des éléments du climat et ceux de l'activité solaire devraient comporter les cycles réels rapprochés.

Les nombres de Wolf, la circulation atmosphérique, la température de l'air et le nombre de jours d'orage présentent le cycle de 11 ans env. On a donc examiné celui-ci en cherchant une dépendance du nombre de jours

\footnotetext{
* Extrait de mémoire de maîtrise préparé sous la direction de Maria Stopa-Boryczka à la Faculté de Géographie et d'Études Régionales de l'Université de Varsovie.
} 
a

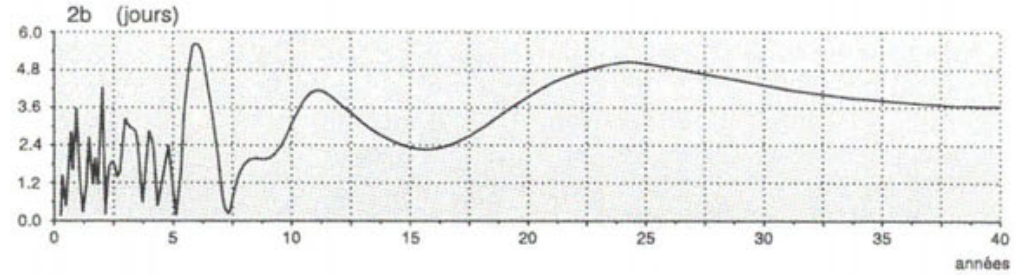

b

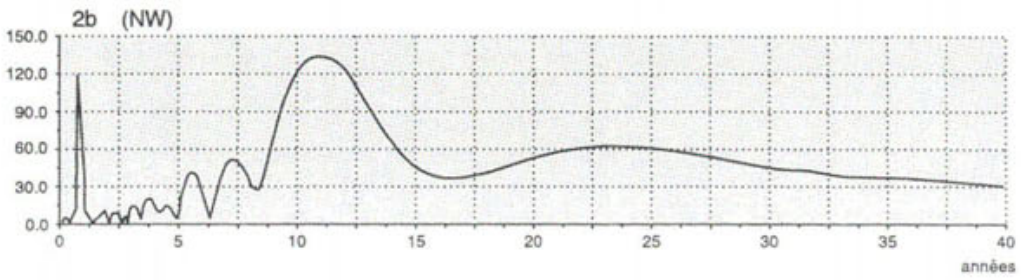

c

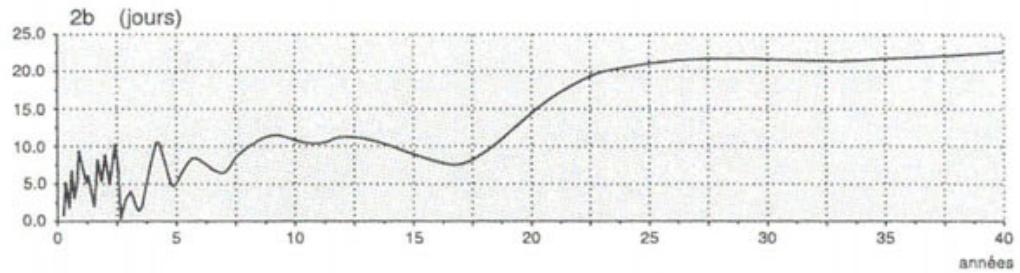

d

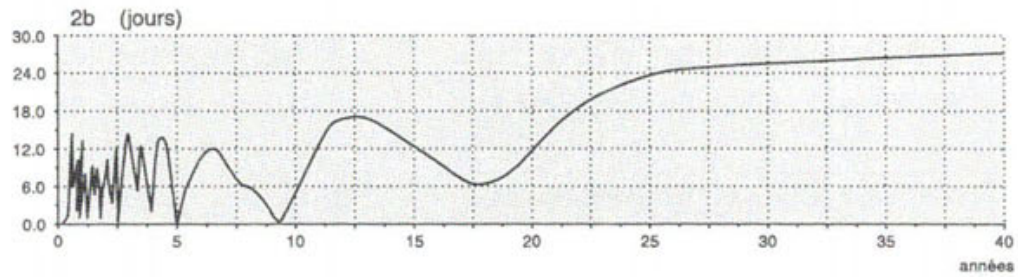

e

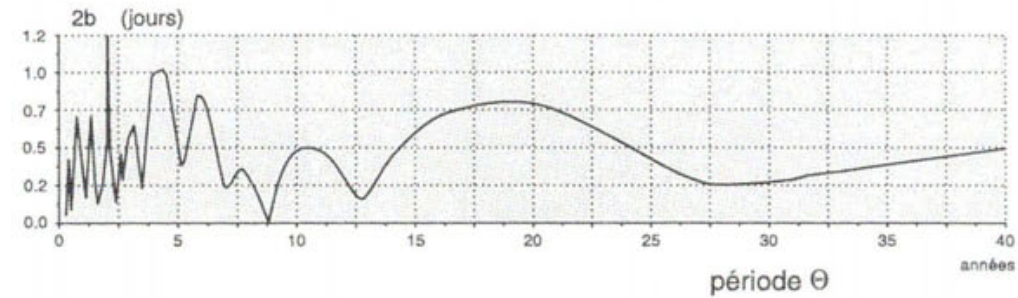

Fig. 1.Le spectre de l'oscillation du nombre de jours d'orage, celui des nombres de Wolf, de la circulation atmosphérique occidentale et orientale et de la température de l'air. Les moyennes consécutives des quatre mois à Varsovie, en 1951-1980 (mai-août): 1a - spectre de l'oscillation du nombre de jours d'orage (1951-1980); moyennes consécutives, $1 \mathrm{~b}$ - spectre de l'oscillation des nombres de Wolf (1951-1980); moyennes consécutives, 1c - spectre de l'oscillation de la circulation atmosphérique occidentale (1951-1980); moyennes consécutives, 1d - spectre de l'oscillation de la circulation atmosphérique orientale (1951-1980); moyennes consécutives, 1e spectre de l'oscillation de la température de l'air à Varsovie (1951-1980); moyennes consécutives, $2 \mathrm{~b}$ - amplitude, $\Theta$ - période réelle. 
d'orage de l'activité solaire, de la circulation atmosphérique (occidentale et orientale) et de la température de l'air.

Parmi les cycles connus des nombres de Wolf, le cycle de 11,08 ans de l'activité solaire était le plus fort (Fig. 1b). L'écart entre les valeurs maximales et minimales dans ce cycle était de 132,9 de nombres de Wolf. Pendant trois décennies examinées, les maxima de ce cycle se sont manifestés en 1957, 1968 et en 1980. Si l'on comparait ces dates avec les maxima du cycle de 11,25 ans du nombre de jours d'orage (Tableau 1, Fig. 1a), on pourrait constater qu'ils sont synchronisés. Les maxima dans le cycle des jours d'orage ont apparu en 1959,1970 et en 1981. Ils sont évidemment déplacés, les uns par rapport aux autres, d'un an ou deux, mais il ne faut pas oublier que le phénomène d'orage dépend d'autres facteurs encore, en dehors de l'activité du Soleil. Seulement l'influence de cette dernière est bien visible, et les maxima du cycle de 11,25 ans suivent toujours immédia-tement les maxima du cycle des taches solaires. Il en est de même en ce qui concerne les dates des minima de l'activité solaire qui se manifestent un an ou deux plus tôt par rapport au cycle de l'activité d'orages. Seulement, lorsque le maximum a été déplacé dans le temps de 1,5 an p.ex., le minimum le serait également de cette même valeur. La comparaison des dates du minimum de l'activité solaire et du minimum de l'activité d'orages est présentée dans le Tableau 1.

Tableau 1

Les années avec les maxima et les minima des nombres de Wolf (NW), du nombre de jours d'orage (NJO), de la circulation orientale $\left(\mathrm{C}_{E}\right)$, de la circulation occidentale $\left(\mathrm{CW}_{W}\right)$ et de la moyenne mensuelle de la température de l'air (T) dans le cycle de 11 ans env

\begin{tabular}{l|c|c|c|c|c}
\hline & NW & NJO & C $_{\mathrm{E}}$ & CW & T \\
\hline Cycles (ans) & 11.08 & 11,25 & 12,5 & 12,33 & 10,42 \\
Années avec les & 1957 & 1958 & 1959 & 1952 & 1951 \\
valeurs maximales & 1968 & 1970 & 1972 & 1964 & 1962 \\
& 1970 & 1982 & 1984 & 1976 & 1972 \\
Années avec les & 1952 & 1953 & 1953 & 1958 & 1956 \\
valeurs minimales & 1963 & 1964 & 1966 & 1970 & 1967 \\
& 1974 & 1976 & 1978 & 1982 & 1972 \\
\hline
\end{tabular}

Les changements qui s'opèrent dans le Système solaire influent sur notre climat par l'intermédiaire de la circulation atmosphérique. Sous les latitudes tempérées, ce sont les circulations occidentale et orientale qui prédominent (Fig. 1c). Une baisse de l'activité solaire renforce le développement de la circulation occidentale tandis que l'accroissement de l'activité du Soleil l'affaiblit (au contraire du cas des jours d'orage). Et c'est ainsi que les maxima dans le cycle de 12,33 ans de la circulation occidentale correspondent aux minima dans le cycle des jours d'orage comme, p.ex., en 1952-1953, 1964 et 1976. Il en est de même dans le cas des dates du minimum de la circulation occidentale qui correspondent à peu près aux dates du maximum du cycle des jours d'orage (Tableau 1). 

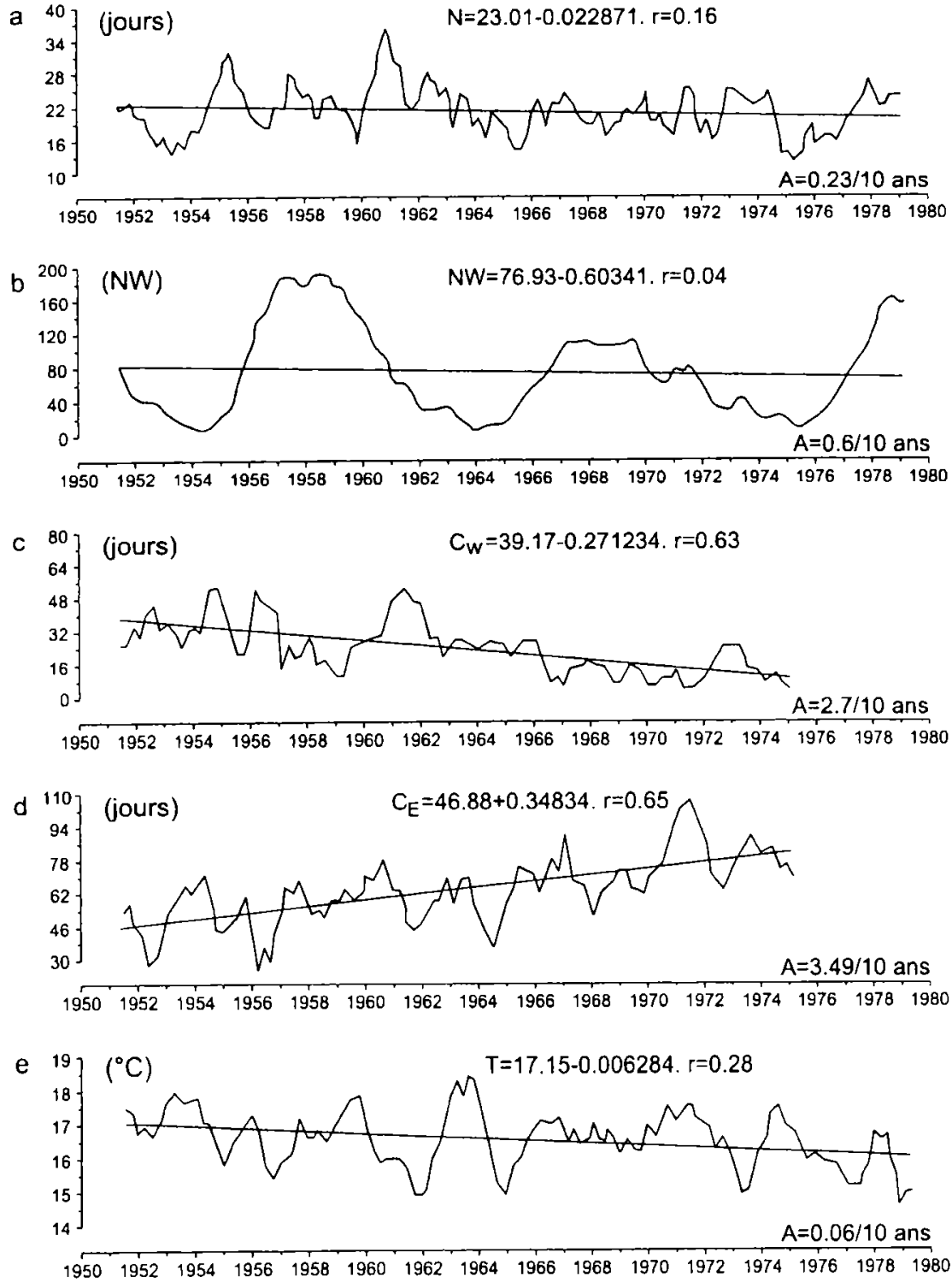

Fig. 2. Les tendances des variations des moyennes consécutives des valeurs de 4 mois calculées pour le nombre de jours d'orage, la température de l'air, les nombres de Wolf et la circulation atmosphérique occidentale et orientale. Varsovie 1951-1980 (mai-aout): $2 \mathrm{a}$ - nombre de jours d'orage; sommes consécutives des 4 mois, $2 b$ - nombres de Wolf; moyennes consécutives des 4 mois, 2c-circulation atmosphérique occidentale; sommes consécutives des 4 mois, $2 \mathrm{~d}$ - circulation atmosphérique orientale; sommes consécutives des 4 mois, $2 \mathrm{e}$ - température de l'air; moyennes consécutives des 4 mois. 
En ce qui concerne la circulation orientale, il y avait également un cycle de 12,50 ans. Cette circulation démontre la plus forte dépendance de l'activité du Soleil et de l'activité d'orages. Aussi bien les maxima que les minima de la circulation orientale qui correspondent à peu près aux valeurs maximales et minimales des jours d'orage sont également déplacés d'un an ou même plus (4 ans) par rapport au cycle des taches solaires (Tableau 1).

Dans le cas de la température de l'air (cycle de 10,42 ans - Fig. 1d), les dates de l'apparition des valeurs maximales et minimales de celle-ci ont 2 ans env. de retard par rapport au cycle des nombres de Wolf (Tableau 1). Il en est ainsi parce que la température de l'air dépend de plusieurs autres éléments, non seulement globaux (dont l'activité solaire, la circulation atmosphérique) mais également locaux (relief du terrain, couverture végétale). Néanmoins, l'interdépendance des cycles des jours d'orage et des cycles de la température de l'air est la plus faible.

On a effectué une comparaison entre les tendances des paramètres examinés dont il a été question plus haut. Au cours des quatre mois choisis (mai-août), le nombre de jours d'orage présente une lente diminution, à savoir presque 2 jours d'orage sur 30 ans (Fig. 2a). La diminution du nombre de jours d'orage est accompagnée de la diminution des nombres de Wolf (2 nombres de Wolf sur 30 ans - Fig. $2 b$ ). Le fait que le nombre de jours d'orage diminuait au cours des trois décennies examinées est dû à une diminution peu importante de la durée de la circulation occidentale (11 jours pendant trente ans) et à un accroissement de la circulation orientale (14 jours pendant 30 ans) dans la circulation atmosphérique générale (Fig. 2c). Tout comme les jours d'orage et les nombres de Wolf, la température de l'air manifeste une tendance décroissante (diminution de $0,3^{\circ} \mathrm{C}$ pendant 30 ans. On observe une alternance des périodes avec les valeurs de la température de l'air tantôt plus tantôt moins grandes (cf. Fig. 2d).

En guise de conclusion, nous aimerions rappeler ici que:

- à l'activité solaire accrue correspond généralement un nombre plus grand de jours d'orage, et à l'activité plus faible - un nombre moins important; c'est donc la plus forte des interdépendances recherchées;

- un accroissement de la fréquence de la circulation orientale est accompagné d'une augmentation du nombre de jours d'orage;

- une apparition de la circulation occidentale en été entraîne une diminution du nombre de jours d'orage. Mais lorsque la circulation occidentale est de caractère cyclonique, le nombre de jours avec les orages d'origine frontale augmente, ce qui est dû à un passage d'une dépression atmosphérique au-dessus du territoire de la Pologne.

Les correlations les plus faibles apparaissent entre l'activité d'orage et la température de l'air. 
